\title{
De l'urgence à représenter le réel de notre monde
}

Entretien de Delphine Edy avec Thomas Ostermeier

The urgency of representing the reality of our world. Interview between

Delphine Edy and Thomas Ostermeier

\section{Delphine Edy}

\section{(2) OpenEdition \\ Journals}

\section{Édition électronique}

URL : https://journals.openedition.org/pratiques/10737

DOI : $10.4000 /$ pratiques. 10737

ISSN : 2425-2042

\section{Éditeur}

Centre de recherche sur les médiations (CREM)

\section{Référence électronique}

Delphine Edy, « De l'urgence à représenter le réel de notre monde », Pratiques [En ligne], 191-192 | 2021,

mis en ligne le 15 décembre 2021, consulté le 04 janvier 2022. URL : http://journals.openedition.org/ pratiques/10737 ; DOI : https://doi.org/10.4000/pratiques.10737

Ce document a été généré automatiquement le 4 janvier 2022.

(c) Tous droits réservés 


\title{
De l'urgence à représenter le réel de notre monde
}

\author{
Entretien de Delphine Edy avec Thomas Ostermeier \\ The urgency of representing the reality of our world. Interview between \\ Delphine Edy and Thomas Ostermeier
}

Delphine Edy

1 Thomas Ostermeier (T.O.), metteur en scène allemand et directeur artistique de la Schaubühne à Berlin, est une véritable étoile du théâtre européen. Ses spectacles tournent dans le monde entier, et pas une saison théâtrale ne s'écoule sans qu'il ne soit à l'affiche en France où son travail est particulièrement apprécié. Artiste associé au festival d'Avignon en 2004, nommé Commandeur de l'Ordre des Arts et des Lettres en 2015, il a reçu le Molière du théâtre public en 2019 pour La Nuit des rois ou tout ce que vous voulez qu'il a créé avec les acteurs de la Comédie-Française. Depuis plus de vingt ans, il défend un théâtre ancré dans le réel, un théâtre engagé qui cherche à « observer la réalité qui nous entoure, c'est-à-dire la réalité du comportement humain, même et surtout dans son caractère contradictoire, et à trouver des formes qui expriment cela sur le plateau ${ }^{1} »$. Dans le cadre d'un numéro qui questionne le réel sur les scènes contemporaines, sa voix était donc fondamentale.

2 Delphine Edy (D.E.), professeure agrégée d'allemand et docteure en littérature comparée, enseigne en classes préparatoires, en études théâtrales et en littérature comparée à l'université de Strasbourg. Spécialiste du théâtre contemporain et en particulier de celui de T. Ostermeier, elle vient de lui consacrer un livre, publié aux presses du réel de Dijon, dont la sortie est prévue au printemps 2022 : Thomas Ostermeier : explorer l'autre face du réel pour recréer l'œuvre en scène.

3 Le texte qui suit est constitué de deux entretiens : le premier a eu lieu à Berlin fin février 2020, à la veille d'un premier confinement mondialisé. Le deuxième, réalisé en juin 2020, a permis de compléter le propos.

4 D.E. : Une chose m'a frappée dernièrement, vous avez cosigné avec É. Louis Au cœur de la violence, et donc adopté le rôle de l'écrivain. Sur cette question de l'écriture, 
j'aimerais revenir sur ce que vous avez dit il y a dix ans en 2009 à l'occasion d'un entretien avec W. Mouawad à Ottawa. À la question "As-tu envie d'écrire des pièces?", vous aviez répondu « Oui, mais je sais que je suis un très mauvais auteur dramatique ». Qu'est ce qui s'est passé entre-temps pour que vous cosigniez un texte publié ? Et est-ce que ça a changé quelque chose pour vous?

T.0.: Dans le cadre de cette collaboration entre É. Louis et moi, je me suis retrouvé dans la situation, non pas forcément d'écrire, mais de chercher des dialogues au sens d'une écriture de plateau, c'est-à-dire au sens où la situation existe déjà, on connait déjà les personnages, on se demande ce que pourrait dire ce personnage et ce que l'autre personnage répondrait, à quoi ressemblerait leur dialogue, comment ils parleraient ensemble. Et puis, j'ai écrit quelques phrases, deux ou trois dialogues et je les ai proposés à É. Louis et ce qui était drôle, c'est qu'il a parfois répondu «ah non, jamais je n'aurais dit ça dans cette situation », « jamais Reda n'aurait dit ça », donc il a compris que, moi, j'avais besoin d'un dialogue, de plus de situations scéniques, et que la prose n'était pas suffisante, et c'est là qu'est née notre collaboration; si je suis capable de faire cela aujourd'hui, cela a beaucoup à voir avec mon travail sur la situation dramatique et la méthode avec laquelle je travaille qui a beaucoup évolué. Grâce à cette évolution, il me semble moins difficile aujourd'hui d'imaginer des dialogues, mais cela reste toujours une sorte de réponse à la situation d'un texte qui existe déjà.

D.E. : Quand vous parlez d'évolution de votre méthode, vous vous référez à l'évolution que décrit par exemple O. Cadiot entre Les Revenants (2013) et La Mouette (2016) ou à une évolution plus récente ou plus large, par exemple au cours des dix dernières années?

7 T.o. : C'est une évolution de chaque jour. Pour vous donner un exemple concret, je sors d'une journée avec les étudiants qui passent le concours de l'École Ernst Busch. On a travaillé sur la dernière scène entre Nina et Treplev dans La Mouette, et je comprends mieux la scène aujourd'hui que lorsque je l'ai montée pour la première fois (Ndlr : à Amsterdam en 2013) et même pour la deuxième fois (Ndlr : en 2016 en France). Je reste dans une sorte d'apprentissage. Il est devenu de plus en plus important pour moi de comprendre comment la méthode de C. Stanislavski évolue; j'ai aussi participé aux stages de M. Bernardin qui travaille avec la méthode de $\mathrm{S}$. Meisner avec des repetition exercises, j'ai fait également deux stages avec S. Batson parce que je voulais savoir ce qu'est le Sense Memory et la méthode de L. Strasberg car la méthode Strasberg, c'est un développement de la méthode Stanislavski. Je m'intéresse de plus en plus au jeu d'acteur et à la manière dont je peux faire fonctionner l'acteur dans une situation dramatique ou dans une situation théâtrale. Cela a aussi à voir avec le fait que, moimême, au cours des trois dernières années, $j$ 'ai joué trois fois dans des films comme acteur, j'ai fait à nouveau des expériences « de l'autre côté » et j'ai compris, qu'avec ma méthode de metteur en scène, j'étais devenu un meilleur acteur. Cela m'a beaucoup aidé car j'adapte ma propre méthode quand je suis acteur pour le cinéma.

Pour en revenir à la question de l'évolution, quand je fais des stages sur W. Shakespeare, je comprends beaucoup mieux les situations dramatiques de la pièce. Je viens de faire la reprise de La Nuit des rois (Ndlr : à la Comédie-Française, février 2020), et à nouveau, je me suis dit, «tu étais vraiment aveugle pendant les premières répétitions ", et ce, qu'il s'agisse de la lecture des scènes ou de celle des personnages, de leurs relations. Dans cette pièce, ils sont toutes et tous amoureux de la mauvaise personne, que ce soit dans les relations hétéro- ou homosexuelles. Prenons par exemple la relation entre Antonio et Sébastien, c'est la relation entre un homme âgé et un jeune 
homme, et ce n'est pas seulement une situation classique au sens grec, mais c'est classique au sens tragique, car, les hommes âgés qui désirent des hommes jeunes et leur beauté, vivent une situation tragique car elle est dans la plupart des cas impossible et, pour Antonio, c'est très douloureux. Au moment du quiproquo, lorsqu'Antonio pense que Sébastien le trahit, avec le terrible constat « il m'aime seulement pour mon argent et non pour ce que je suis ", on assiste à une sorte de nouvelle réflexion au sens d'une réfraction (Spiegelung) des autres relations de la pièce (Olivia et Cesario, Orsino et Olivia, Orsino et Cesario) : cette relation n'est pas seulement une relation comique mais profondément tragique, car cela parle aussi des amours impossibles en lien avec la question du pouvoir (le pouvoir sur l'autre, le pouvoir lié à l'argent), qui, après celle de l'amour et de la mort, est le thème majeur chez W. Shakespeare. Dès les premières représentations, on avait choisi de ne pas camoufler, comme c'est parfois le cas, le fait qu'il s'agisse d'une relation homosexuelle, mais la lecture n'était pas assez profonde, car il manquait l'épaisseur de la différence d'âge et aussi celle du pouvoir lié au fait que Sébastien est un naufragé, totalement perdu, sans argent et qui n'a pas d'autre choix que de s'en remettre à Antonio, même si son but principal est de se libérer de cette situation de dépendance. Ce n'est donc pas un simple "jeu » de séduction entre deux homosexuels qui se flattent mais bien un jeu de pouvoir entre eux.

Voilà, ce sont ces choses qui évoluent et que j'ai essayé de décrire dans The Theater of Thomas Ostermeier ${ }^{2}$. Dans la lignée de C. Stanislavski, je fais une différence entre la situation et la situation dramatique, donc la situation est la somme des circonstances que l'on connait mais aussi celle qui fait agir les personnages. Il y a un besoin de la situation dramatique qui fait qu'il faut agir, sinon, on est mort, malheureux, pauvre. J'appelle cela des zwingende Handlungsumstände, des circonstances qui font qu'il faut agir dans la situation. Je n'en parle pas encore en ces termes dans The Theater of Thomas Ostermeier et c'est ce qui est devenu très important pour moi ces cinq dernières années, on a beaucoup travaillé à partir de ce diptyque "situation et situation dramatique " pour La Nuit des rois. Un autre exemple: nombreux sont ceux qui se demandent pourquoi Viola se déguise en homme au début de la pièce. Et plusieurs metteurs en scène m'ont dit avant que je ne commence le travail, « c'est une belle pièce, mais il n'y a pas de réponse à cette question ", et pour moi, pourtant, c'est évident : elle est forcée de se travestir car elle vit dans un monde médiéval où une femme indépendante n'a pas le choix, car seule la prostitution pourrait sinon la faire survivre. Dans cette société patriarcale, elle n'a pas le choix, c'est une force qu'elle ne peut que contourner par le déguisement. Et aujourd'hui encore, on vit dans un système patriarcal, donc on peut comprendre cette décision même si aujourd'hui on pourrait trouver d'autres solutions, mais la métaphore fonctionne encore très très bien.

D.E. : À propos de la situation dramatique, j'aimerais qu'on s'attarde un moment sur la question du langage: quels sont les mots que l'on peut dire? Quel est le sens du langage?

11 T.O.: Sprache ist Handlung [Le langage est action]. Donc, dans une pièce dramatique, chaque phrase est une action au sens de Sprechakt, avec laquelle j'essaie de faire bouger mon adversaire, mon partenaire, mon interlocuteur. La situation dramatique part d'un conflit que j'ai avec quelqu'un d'autre, mais je suis bien obligé de partager mon monde avec cette personne, et il devient donc absolument nécessaire de trouver un accord. Ce n'est que dans un monde partagé qu'il y a la nécessité de trouver des solutions. C'est pour cela que la plupart des pièces sont des pièces sur la famille. Cela étant, chez 
W. Shakespeare, le moment du dialogue dramatique, c'est le moment qui précède le fait de sortir son épée. Et donc, si l'on ne parvient pas à trouver une solution avec l'arme du langage, il ne reste qu'à utiliser la vraie force physique et c'est ce qui crée la dimension dramatique : il s'agit alors de la vie ou de la mort.

D.E. : L'arme de la langue, c'est aussi ce qui est au cœur du spectacle que vous avez présenté à Paris en février 2020 avec É. Louis, Qui a tué mon père. C'est l'arme qu'il utilise pour exister lui-même et reconstruire la relation à son père. Par ailleurs, la langue vous permet également de présentifier l'absence du père sur scène ; elle a donc le pouvoir de faire exister l'invisible.

T.0. : C'est la langue, vous avez raison, mais c'est aussi le jeu d'É. Louis. Il a suivi mes indications. Je lui ai demandé de s'imaginer que son père était véritablement présent sur scène, car, au début, il traitait le langage de manière très classique, au sens classique français, c'est-à-dire de manière très déclamatoire. Dans ce spectacle, la situation dramatique, c'est celle de la classe ouvrière, de la classe populaire, des exclus, des gens de province, des gens marginalisés, qui existent aussi en Allemagne d'ailleurs, et c'est ce qui fait qu'il y a une sorte d'urgence à ce que cela soit représenté, au sens double du terme d'ailleurs : que ce soit à la fois représenté sur scène, dans la littérature, au cinéma, à la télévision, dans la fiction et dans le réel, et que cela soit représenté politiquement. Il y a un lien entre les deux et c'est beau que ce double sens existe dans la langue française et dans la langue anglaise, ce n'est pas le cas en allemand, die Repräsentation, c'est toujours politique. Et je trouve cela très beau car c'est l'un des problèmes les plus sérieux et les plus frustrants de notre situation aujourd'hui avec l'extrême-droite qui prend le pouvoir, car tous ces gens ne sont pas représentés, au sens politique (à cause de la faiblesse de la gauche, tout ce que décrit en fait Didier Eribon dans Retour à Reims au cours des trente dernières années) et à cause de cette faiblesse, à cause de cette trahison de cette mission historique de la gauche, il n'y a plus de représentation sauf la représentation raciste, homophobe et nationaliste de l'extrême-droite.

D.E. : J'ai le sentiment que vous avez bien changé depuis le moment où vous parliez avec W. Mouawad, comme si écrire devenait davantage possible?

T.0.: Oui, c'est vrai, car je suis un grand fan de cinéma. J'aime davantage le cinéma que le théâtre et quand je vois la faiblesse de la dramaturgie de certains films, la faiblesse du storytelling, je me dis qu'il faut changer cela : il y a trop de films où il n'y a pas de drame dans toute sa complexité et ses contradictions, alors que les vrais films, ceux qu'on aime et qui restent en mémoire, ce sont ceux plein de contradictions. Je crois que la force de la littérature et des grands textes de théâtre, c'est la force de pouvoir raconter une histoire. Depuis que les hommes sont sur terre, ils essaient de comprendre leur situation, ce que cela signifie d'être un être humain, ils essaient de trouver des structures et de l'ordre dans le chaos de notre existence. Il est bien sûr impossible de comprendre pourquoi on est là et pourquoi on meurt à un moment, il n'y a aucune raison à notre existence, mais on essaie de trouver un sens, une structure et un ordre : c'est le cas avec l'ordre divin, avec la perspective d'une deuxième vie, mais aussi avec la philosophie, la politique et la sociologie. Ce sont à chaque fois des essais pour donner du sens et comprendre ce qu'est un être humain et la société. La littérature, lorsqu'elle raconte des histoires, n'a pas besoin de donner du sens à tout ce qu'il se passe. Mais en philosophie, en politique, en sociologie, tout doit faire sens et tout doit pouvoir être déchiffré pour donner du sens à la réalité. Or, on ne peut pas tout comprendre, donc le 
théâtre ou le roman, c'est la meilleure réflexion (là encore au sens de réfraction) du monde, car c'est une réflexion du monde avec toutes les contradictions qui font notre existence.

D.E. : Il me semble que votre travail jusqu'à aujourd'hui, que ce soit dans des mises en scènes de textes classiques (W. Shakespeare, H. Ibsen, A. Tchekhov, A. Schnitzler, Ö. von Horváth ...) ou de textes contemporains, montre que vous êtes à la fois complètement dans le réel, dans l'exactitude des rapports entre les gens, dans leurs histoires ... et en même temps que vous le mettez à distance. En tant que spectatrice, je suis à la fois complètement dans la représentation du réel, je me trouve immergée, et, en même temps, j'ai accès à une autre face du réel, son « double » en quelque sorte.

17 T.O. : Je suis d'accord sur le fait que, dans mes spectacles, il y a une grande partie où les conflits et les comportements des gens et les relations entre les personnages sont de nature réaliste, mais en même temps, c'est aussi vrai qu'il y a une autre dimension dans mon travail - c'est toujours difficile de parler de son propre travail - au sens où j'essaie de surmonter cette réalité dans une autre dimension, une dimension où d'un coup j'ouvre le crâne du personnage et je fais en sorte de descendre jusqu'à l'inconscient du personnage, dans les images sur scène, dans les chorégraphies... Dans Personenkreis 3.1., où il est question de drogués, de sans-papiers, d'exclus, il y a une scène avec un héroïnomane qui s'injecte une dose et elle s'enchaine avec une chorégraphie en musique qui vise à surmonter l'image réaliste (celle d'un drogué amorphe, tête baissée et bras ballants) et essaie de décrire ce qu'il se passe à l'intérieur de cet homme.

D.E. : Vous faites allusion à Personenkreis 3.1 de L. Norén, mais cela me fait aussi penser à votre Woyzeck que j'ai pu découvrir sur le site de la Schaubühne pendant le confinement qui me semble être un condensé de plein de choses que vous allez développer plus tard.

T.O. : Oui, et en plus c'était juste avant les émeutes en banlieue parisienne en 2005...

D.E. : Tout à fait, comme si le théâtre avait cette capacité de voir par devant le réel. Ce que vous dites à propos de Personenkreis 3.1, fait également écho à ce que vous avez proposé dans Abgrund de M. Zade (2019), où on a ces images vidéo en contre-plongée qui nous donnent accès à une autre facette du personnage, à ce qui se passe à l'intérieur de lui. On peut dire ça?

T.0.: Oui, et on peut dire, je crois, que l'on trouve de tels moments dans tous mes spectacles, plus ou moins.

D.E. : On est d'accord.

T.0. : Même dans Retour à Reims, dans la deuxième partie du film, il y a le moment avec la chanson de G. Scott-Heron sur les images de mai 68 et celles du Vietnam où j'essaie de donner une image de l'esprit du temps, à la fois cette violence et en même temps le rock'n'roll; ça se retrouve dans ce documentaire, car c'est influencé par un documentariste anglais que j'adore, A. Curtis, qui a fait un film HyperNormalisation, qui n'est pas un documentaire «pur » qui essaie de rester sobre et juste, c'est plutôt une sorte de sampling [échantillonnage], comme un trip LSD.

D.E. : Encore une question à propos de la représentation des exclus, des marginaux, des pauvres, des gens qui ne sont pas reconnus par la société. Je me suis demandé si c'était un choix délibéré de monter ces textes après avoir monté Ö. von Horváth et A. Schnitzler : car il y a pour moi ici un dialogue entre Ö. von Horváth et A. Schnitzler qui décrivent des mécanismes concrets - comment le pouvoir s'installe, comment se construit cette relation de pouvoir et comment l'antisémitisme (présent depuis le 
Moyen Âge) et l'idéologie national-socialiste prennent le dessus - et D. Eribon et É. Louis qui eux représentent les victimes de ces mécanismes de pouvoir.

T.0.: Je n'y ai pas pensé du tout, en fait, c'est plutôt moi qui ai un certain point de vue marxiste, au sens où «das gesellschaftliche Sein bestimmt das Bewusstsein » [l'être social détermine la conscience] et c'est mon combat personnel car aujourd'hui, la plupart des mouvements, sont des appels à l'idéalisme. Même l'appel que j'ai cosigné dans Le Monde était un appel à l'idéalisme avec l'idée que maintenant on a bien vu qu'il était possible d'arrêter la machine capitaliste pour faire mieux, donc saisissons cette expérience pour faire mieux après sur le plan écologique, climatique etc... Je suis d'accord avec cela bien sûr, mais je n'y crois pas. Je signe bien évidemment car je suis complètement pour mais je ne pense pas qu'un appel d'artistes et d'intellectuels va changer le monde, je suis beaucoup trop marxiste pour y croire. Et être marxiste, cela veut dire être un matérialiste historique, au sens où la situation matérielle fait la vie des gens. Et tous les textes que je monte, je les regarde avec de telles lunettes. Mais il faut se méfier car aujourd'hui les lunettes matérialistes sont souvent comprises comme des lunettes capitalistes (où le matériel compte plus que tout), car les gens ne savent pas que les lunettes matérialistes sont celles de K. Marx. Jusqu'au moment où j'ai monté H. Ibsen en 2002 avec Nora, il n'y avait jamais eu de lecture de gauche de H. Ibsen, on le voyait comme l'écrivain de la bourgeoisie, de la tristesse de la grande bourgeoisie, avec la psychologie, l'âme comme paysage, les salons etc... Mais quand j'ai lu H. Ibsen, j'ai compris que c'était l'argent qui était au cœur de sa dramaturgie : c'est vrai dans Hedda Gabler, dans John Gabriel Borkmann, dans Maison de poupée, il y a toujours cette inquiétude, cette peur de ne pas réussir dans la société bourgeoise. Quand je m'approche de Ö. von Horváth mais aussi de F. X. Kroetz ou de A. Schnitzler, c'est mon regard qui essaie de bien lire ces textes et d'analyser profondément. Et avec cette analyse de peut-être voir de nouvelles perspectives sur un texte canonique. Il y a des textes fortement marxistes qui ne m'intéressent pas car, un de mes principes les plus importants, c'est celui du contre-point, donc c'est mieux de regarder une œuvre canonique classique avec des lunettes de l'extrême-gauche que de regarder du B. Brecht, parce que c'est un «weißer Schimmel » [l'exemple parfait de la tautologie, c'est-à-dire dire la même chose]. Donc il n'y a pas forcément un lien, une sorte de logique entre mes choix de textes, c'est peut-être davantage de l'ordre du hasard : il y a le texte à un moment parfait où j'ai du temps libre, des acteurs, l'envie, il y a l'urgence de le mettre en scène, donc je le fais. Mais deux ans plus tard, il n'y a plus d'urgence. C'est plutôt l'urgence qui me pousse à faire des choix, la logique m'importe peu.

D.E. : Je crois aussi que c'est votre point de vue sur le monde qui s'exprime dans le choix des œuvres que vous montez et que vous le vouliez ou non, forcément les œuvres de A. Schnitzler et Ö. von Horváth, dans la manière dont vous les traitez, dialoguent avec celles de D. Eribon et É. Louis. C'est plutôt comme cela qu'il faudrait dire les choses?

T.O. : Oui, tout à fait.

D.E. : Pour en revenir à la manière dont vous traitez le réel, il y aurait donc une « face » et son " double ", au sens spectral de J. Derrida, qui viendrait hanter le réel, et c'est ce qui est très beau quand il y a toutes ces zones d'ombres (au travers de la situation dramatique, au travers de l'utilisation de la musique, de la vidéo). Ce qui m'a particulièrement interpelée, c'est l'écho - dans le traitement de l'image - que vous 
faites à Retour à Reims dans Qui a tué mon père. C'est un écho interne qui raconte toute une histoire, mais qui raconte aussi le lien entre ces deux histoires.

T.O. : Oui, absolument, et qui raconte aussi le lien entre É. Louis et D. Eribon en tant que son mentor, et on perçoit dans tous les textes d'É. Louis des traces de Retour à Reims, on voit très très bien qu'il a comme inhalé ce livre, il habite dans Retour à Reims (Er bewohnt diesen Text von Didier).

D.E. : Il y a en fait une forme de hantise de D. Eribon dans les textes d'É. Louis, une dimension spectrale.

31 T.o.: Oui, mais est-ce qu'on peut vraiment aimer un spectre ? Car je pense qu'il aime vraiment $\mathrm{D}$. Eribon, à la fois comme personne et comme maitre, c'est vraiment une histoire d'amour entre eux.

D.E. : Vous avez raison, il y a peut-être quelque chose de trop inquiétant dans le verbe " hanter " alors que je ne le prenais pas du tout comme quelque chose de négatif. Bien au contraire. Donc il vaut surement mieux retourner la chose en parlant d'habiter, je suis d'accord. Il y a encore une chose sur laquelle je voudrais revenir : vous avez dit que vous n'étiez pas un auteur, pas un artiste, mais un artisan. Et je ne suis pas du tout d'accord et j'aimerais comprendre pourquoi vous refusez ce mot.

T.O.: J'ai trop d'admiration pour de vrais artistes.

D.E. : Car vous n'êtes pas un vrai artiste?

T.O. : Non.

D.E. : Je pense au contraire, que vous êtes un véritable auteur, au sens étymologique de celui qui augmente et c'est très fort dans le travail sur Retour à Reims. Le théâtre agit dans ce cas comme une augmentation de la réalité et ça revient à ce que l'on disait juste avant : cette réalité a besoin d'être augmentée pour trouver son véritable sens et pour renvoyer au spectateur ce qu'ils n'ont pas forcément vu en lisant les livres. On a accès à une forme d'invisible sur la scène et c'est ce qui est très beau.

37 T.0.: Je suis d'accord avec tout ce que vous décrivez, l'augmentation de la réalité, peutêtre, mais c'est lié au hasard, ce n'est pas mon but dans le travail.

D.E. : Mais ce n'est pas ce que je disais non plus. Quand j'analyse votre travail, que vous le vouliez ou non, que ce soit conscient ou non, à la fin, quand on est spectateurs et qu'on voit vos mises en scène, on ne peut être que sensible à cela et c'est ce qui fait la force d'un spectacle.

T.O. : Je suis tout à fait d'accord avec vous, car cela m'arrive aussi parfois. Quand je n'ai pas vu un spectacle depuis longtemps, d'un coup je comprends quelque chose sur le texte que je n'avais pas compris avant. Moi-même j'ai des moments de Haha-Erlebnisse, des moments de " ah oui », et cela m'arrive surtout avec les textes de W. Shakespeare, car ce sont les plus complexes et les plus profonds, mais ça peut arriver aussi avec des textes plus contemporains.

D.E. : Vous arrivez donc à vous surprendre vous-même?

T.O.: Theater ist Denken im dreidimensionalen Raum [le théâtre, c'est penser dans un espace en trois dimensions] et moi, j'aime réfléchir en trois dimensions. La présence d'une troisième personne au théâtre crée un triangle et ça veut dire qu'il y a bien une troisième vérité dans notre système binaire. Or, la troisième réalité est complètement refoulée, elle n'existe plus dans notre monde en deux dimensions (livre, télévision, ordinateur...). La troisième possibilité est pourtant extrêmement importante, par 
exemple la non-existence d'un genre entre les deux genres, c'est quelque chose qui me passionne en ce moment. Quand on fait des recherches là-dessus, on s'aperçoit que dans d'autres cultures, ce troisième genre était un état de fait, un fait connu, un fait considéré comme naturel. Voir apparaitre une constellation de différents personnages sur le plateau, cela apporte de la profondeur et du sens. Dans les répétitions, j'essaie même si mon théâtre a toujours l'air d'être du théâtre allemand dans le mauvais sens, un théâtre sec, précis (genau), et ordonné (ordentlich), comme si j'étais obsédé par l'exactitude donc peut-être trop académique, c'est le danger - de faire agir le hasard dans mes propres mises en scène, et c'est assez difficile.

D.E. : C'est incroyable de vous entendre dire cela, car c'est très exactement la chose qui m'a stupéfaite quand j'ai vu Othello, «mon premier Ostermeier » en 2011 ; c'était à la fois très précis et terriblement juste, mais il y avait aussi une espère d'énergie venue d'ailleurs que j'ai eu envie de questionner: qu'est-ce que c'est? comment cela fonctionne?

T.O. : Cela a à voir avec ma phrase préférée d'A. Artaud qui dit : « Man muss das Chaos in eine tosende Ordnung bringen » [ " Il faut que tout / soit rangé / à un poil près / dans un ordre fulminant ${ }^{3}$. $\left.)\right]$ J'adore cette phrase car c'est absolument ma mission. Dans notre réalité médiatisée, dans une réalité où la plupart de nos communications se passent au travers d'un écran (via un smartphone ou un ordinateur), il faut se méfier des images, des vidéos, il faut se méfier des faits écrits, des fake news, des fake fake news, de la notion même de fake news, donc ce qu'il se passe devant moi dans un espace tridimensionnel, c'est le dernier endroit où l'on ne peut pas tricher. Et l'acte théâtral, l'acte de l'acteur, est un acte qui est encore virginal ; tous les autres médias ont perdu leur virginité. Et les spectateurs ont envie de cet endroit où ils ne sont pas trompés, où il existe une vérité du moment, car ce n'est pas seulement la vérité du corps dans l'espace, c'est aussi la vérité de l'acte créatif, car l'acteur doit être créatif dans le moment sur scène et s'il n'y arrive pas, il échoue. Si tu fais un film, tu fais plusieurs prises et puis tu choisis, et au montage, tu construis quelque chose qui n'a pas beaucoup à voir avec le travail scénique. Tout cela n'est pas possible au théâtre. Donc digitalize the reality, digitalize the world (numériser la réalité, numériser le monde), cela rend service au théâtre, parce que les gens ont un grand désir d'un endroit partagé avec les autres spectateurs et autour d'un événement qui se passe ici et maintenant et plus jamais après, et qui n'existait pas avant. Et le fait que le théâtre soit éphémère, cela en fait une très belle métaphore de la vie, car à la fin, il y a justement une fin.

D.E. : Vous dites souvent que vous n'avez rien à voir avec le postdramatique mais dans cette manière que vous avez de déconstruire le réel pour le reconstruire, vous jouez beaucoup avec la dimension performative des acteurs, vous jouez en fait sans cesse avec les codes de la performance.

T.0. : Oui, mais toujours dans le cadre d'une narration. C'est très complexe, car il s'agit d'abord du travail d'acteur. Je pense que le postdramatique et peut-être plus encore le théâtre des experts du quotidien, c'est le résultat d'une crise du travail d'acteurs, car ils ne sont plus capables d'incarner des personnages du réel. Et cela a à voir avec une crise de formation des acteurs, mais aussi du manque d'humilité, au sens où ils ne se demandent pas suffisamment quelle est exactement la situation du personnage, sa vie, ses circonstances de vie et c'est pour cela que c'était un grand bonheur de travailler avec É. Louis car il est le plus grand expert de son propre texte. Il a fait le travail de l'acteur à partir de sa vie et donc il était à la fois l'auteur, le dramaturge, le personnage 
et le penseur politique qui voulait exprimer quelque chose sur la situation actuelle du monde. C'est un activiste politique qui a fait son homework d'acteur en interrogeant la complexité de son propre personnage puisqu'à la fin, il termine sur une déclaration d'amour à son père après avoir dit pendant longtemps qu'il le détestait. La situation du théâtre postdramatique et le fait de mettre en question les acteurs, c'est le résultat d'une crise de formation et de vie quotidienne des acteurs car leur cadre de référence, ce n'est pas la réalité mais le théâtre ou le cinéma. Alors ils essaient de créer des effets qui servent à leur carrière, sur scène, devant la caméra, mais ce n'est plus le réel ; leur point de référence, ce n'est plus le réel, mais leurs collègues, les spectateurs, les critiques, mais jamais le réel, ce qu'ils ont vu dans le métro, dans leur vie, dans la rue. Et c'est ce qui explique ma grande fascination pour les écrivains contemporains, pour É. Louis et D. Eribon par exemple, car ils regardent autour d'eux et ne répètent pas les clichés. Ils voient et essaient de décrire ce qu'ils voient avec leurs outils.

\title{
NOTES
}

1. Ostermeier, T. (2016). Le Théâtre et la Peur. Arles : Éd. Actes Sud, p. 64.

2. Ostermeier, T. \& Boenisch, P. (2016). The Theatre of Thomas Ostermeier. Londres/New York : Routledge.

3. Phrase notée sur le haut de la première page (comme une indication musicale à côté de la portée), mais qui ne sera pas prononcée dans l'émission transcrite dans Artaud A. (2003). Pour en finir avec le jugement de dieu suivi de Le Théâtre de la cruauté. Paris: Gallimard, p. 23.

\author{
AUTEUR \\ DELPHINE EDY \\ Université de Strasbourg, Accra, F-67081 Strasbourg, FranceSorbonne Université, CRLC, F-75006 \\ Paris, France
}

